\title{
Cervical Myelopathy Caused by Disc Herniation at the Segment of Existing Osteochondroma in a Patient with Hereditary Multiple Exostoses
}

\author{
Ko Ikuta ${ }^{1}$, Kiyoshi Tarukado ${ }^{2}$, Hideyuki Senba ${ }^{1}$, Takahiro Kitamura ${ }^{1}$, \\ Norihiro Komiya ${ }^{1}$, Satoshi Shidahara ${ }^{1}$ \\ ${ }^{1}$ Department of Orthopedic Surgery, Karatsu Red Cross Hospital, Karatsu, Japan \\ ${ }^{2}$ Department of Orthopedic Surgery, Kyushu University Beppu Hospital, Beppu, Japan
}

Hereditary multiple exostoses (HME) is a benign hereditary disorder characterized by multiple osteochondromas. Osteochondroma appears occasionally in the spinal column as a part of HME. A 37-year-old man presented with a history of HME and cervical compressive myelopathy caused by intraspinal osteochondroma arising from the lamina of the C5 and disc herniation at the C5-6. He was treated by open-door laminoplasty at the $\mathrm{C} 5$ and $\mathrm{C} 6$ with excision of the tumor. The neurological symptoms were immediately relieved after surgery. Magnetic resonance images demonstrated a sufficient decompression of the spinal cord with a spontaneous regression of the herniated disc at one year after surgery. There was no recurrence of the tumor and no appearance of kyphosis and segmental instability of the cervical spine on postoperative imaging studies for three years after surgery. The patient could be successfully treated by laminoplasty with excision of the tumor and without removal of the herniated disc.

Keywords: Herniated disc; Osteochondroma; Hereditary multiple exostoses; Decompression; Regression

\section{Introduction}

Spinal osteochondroma is a rare phenomenon, and can originate as a solitary lesion or as a part of hereditary multiple exostoses (HME). HME is a benign hereditary disorder characterized by multiple osteochondromas usually affecting the metaphysical regions of long bones; osteochondromas arise occasionally in the spine. Although cervical spinal cord compression due to intraspinal osteochondroma in HME is a rare condition, there have been more than fifty reported cases of cervical involvement of HME, including 48 cases summarized as a review of literature by Lotfinia et al. [1] in 2010 [2-9]. However, to the best of our knowledge, there have been no previous reports of cervical spinal cord compression caused by disc herniation and intraspinal osteochondroma at the same segment in a patient with HME. This is the first report of a patient of HME with cervical myelopathy caused by disc herniation at the segment of existing intraspinal osteochondroma.

\section{Case Report}

A 37-year-old man with a history of HME was admitted to Karatsu Red Cross Hospital with complaints of a 6-week history of gradually worsening numbness in

Received Sep 22, 2013; Revised Nov 3, 2013; Accepted Nov 13, 2013

Corresponding author: Ko Ikuta

Department of Orthopedic Surgery, Karatsu Red Cross Hospital, 1-5-1 Futago, Karatsu, Saga 847-8588, Japan

Tel: +81-955-72-5111, Fax: +81-955-73-9530, E-mail: kou-ikuta@karatsu.jrc.or.jp 
bilateral hands and feet. The motion of the cervical spine irritated pain in the right lower extremity. His father also had had HME.

Physical examination revealed multiple palpable exostoses in the lower extremities (Fig. 1). On neurological examination, hypesthesia below the T6 dermatome on the right and the $\mathrm{C} 7$ and $\mathrm{C} 8$ dermatomes on the left were demonstrated. There were bilateral hypoactive triceps tendon reflexes and hyperactive deep tendon reflexes in the right lower extremity, and a Babinski response was elicited on the right. No weakness of extremities strength was observed. The patient's Japanese Orthopaedic Association (JOA) score was 12 points.

On plain radiographs (X-rays), an osteochondroma arising from the lamina of $\mathrm{C} 5$ was suspected. Computed tomography (CT) showed a bony outgrowth from the anterior surface of the $\mathrm{C} 5$ lamina on the right with growth into the spinal canal anteriorly (Fig. 2A, B). Magnetic resonance image (MRI) demonstrated an extradural mass arising from the lamina of $\mathrm{C} 5$ on the right and a herniated disc on the left. The spinal cord was severely compressed anteroposteriorly in the shape of a gourd (Fig. 2C, D).

The patient underwent an open-door laminoplasty at the C5 and C6 with excision of the tumor. The operative findings showed a dural tube that was severely compressed by the bony lesion arising from the lamina of C5. The bony lesion, in which a cartilage cap and osseous stalk extended into the spinal canal, was completely en bloc removed by a hemilaminectomy of the $\mathrm{C} 5$ on the

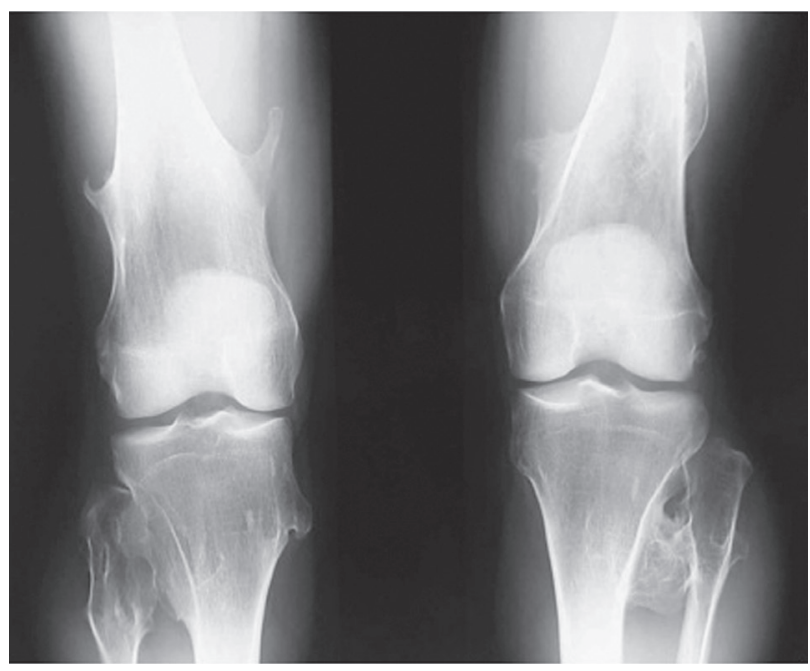

Fig. 1. Plain radiograph of lower extremities showing exostoses. right (Fig. 3A). The opened laminas of C5 and C6 were reconstructed using hydroxyapatite spacers. A small hole was made at the lateral mass and opened lamina to allow passage of a non-absorbable suture from the spacer at each site and the spacer was sutured tightly for bridging between the opened lamina and lateral mass (Figs. 3B, 4). Removal of the herniated disc was not performed.

The histological examination confirmed the lesion to be an osteochondroma. The clinical symptoms were immediately relieved after surgery. The patient was discharged on the 16th day after surgery; he returned to work four weeks after surgery.

Although the patient complained of mild numbness in the right hand and foot, he had no restriction of daily activity and his work one year after surgery. On MRI evaluation, a sufficient decompression of the spinal cord with a spontaneous regression of the herniated disc at the C5-6 was observed one year after surgery (Fig. 5). The patient's JOA score improved four points from the pre-surgery
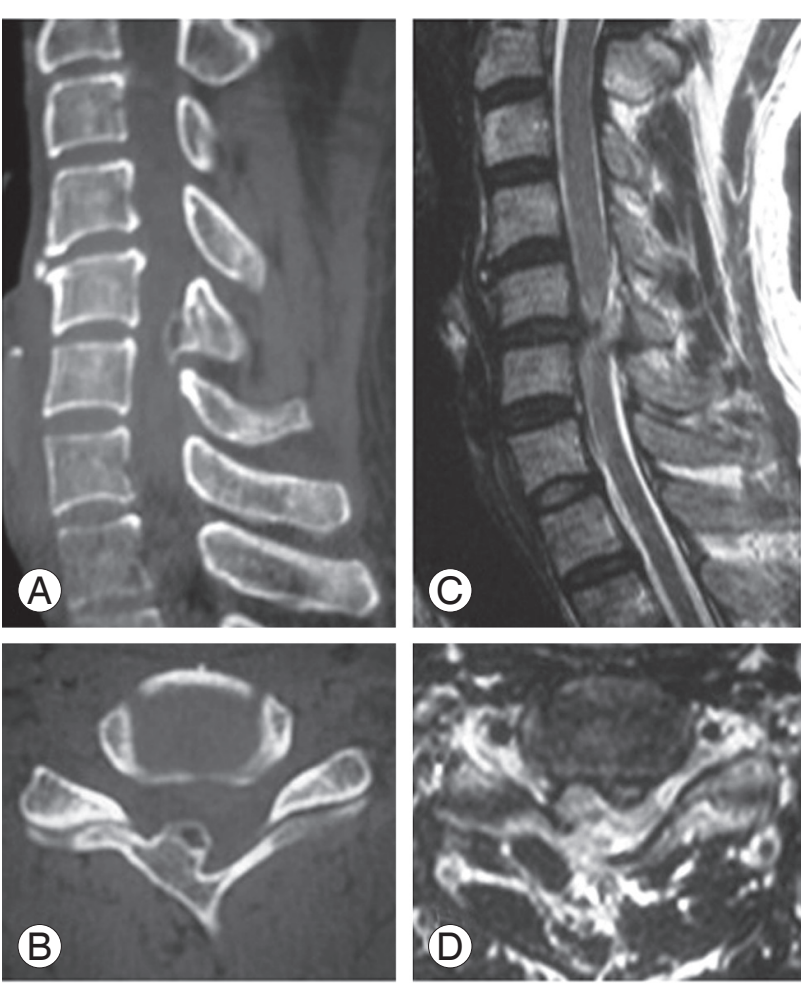

Fig. 2. (A, B) Sagittal and axial computed tomography scan showing the osseous tumor arising from the anterior surface of the C5 lamina on the right with growth into the spinal canal anteriorly. (C, D) Sagittal and axial T2-weighted magnetic resonance images revealing an extradural lesion that compressed the spinal cord anteriorly on the right and a herniated disc that compressed the spinal cord posteriorly on the left. 
level of 12 to 16 points at the three-year follow up. Plain radiographs showed no appearance of the kyphosis and segmental instability of the cervical spine and there had been no recurrence of the tumor at the latest follow-up (Fig. 6).

\section{Discussion}

Spinal osteochondroma is a rare phenomenon and can originate as a solitary lesion or as a part of HME. Although the incidence of spinal osteochondroma in HME had been estimated at approximately $3 \%$ to $9 \%$ of patients with HME [1,10], Roach et al. [7] reported that thirty
(68\%) of the forty-four patients with HME had exostoses arising from the spinal column and twelve (27\%) had lesions encroaching into the spinal canal in 2009. They concluded that the risk a patient with HME had a lesion within the spinal canal is much higher than previously suspected. Spinal involvement of HME usually appears in the second and third decade of life $[1,10]$. Spinal osteochondroma can arise from any part of the spine, but the cervical spine is most commonly involved $[1,10]$. The increased rate of spinal osteochondroma arising from the cervical spine may be attributed to the increased mobility of the cervical spine, which could lead to greater stress and microtrauma to the epiphyseal cartilage, with subse-
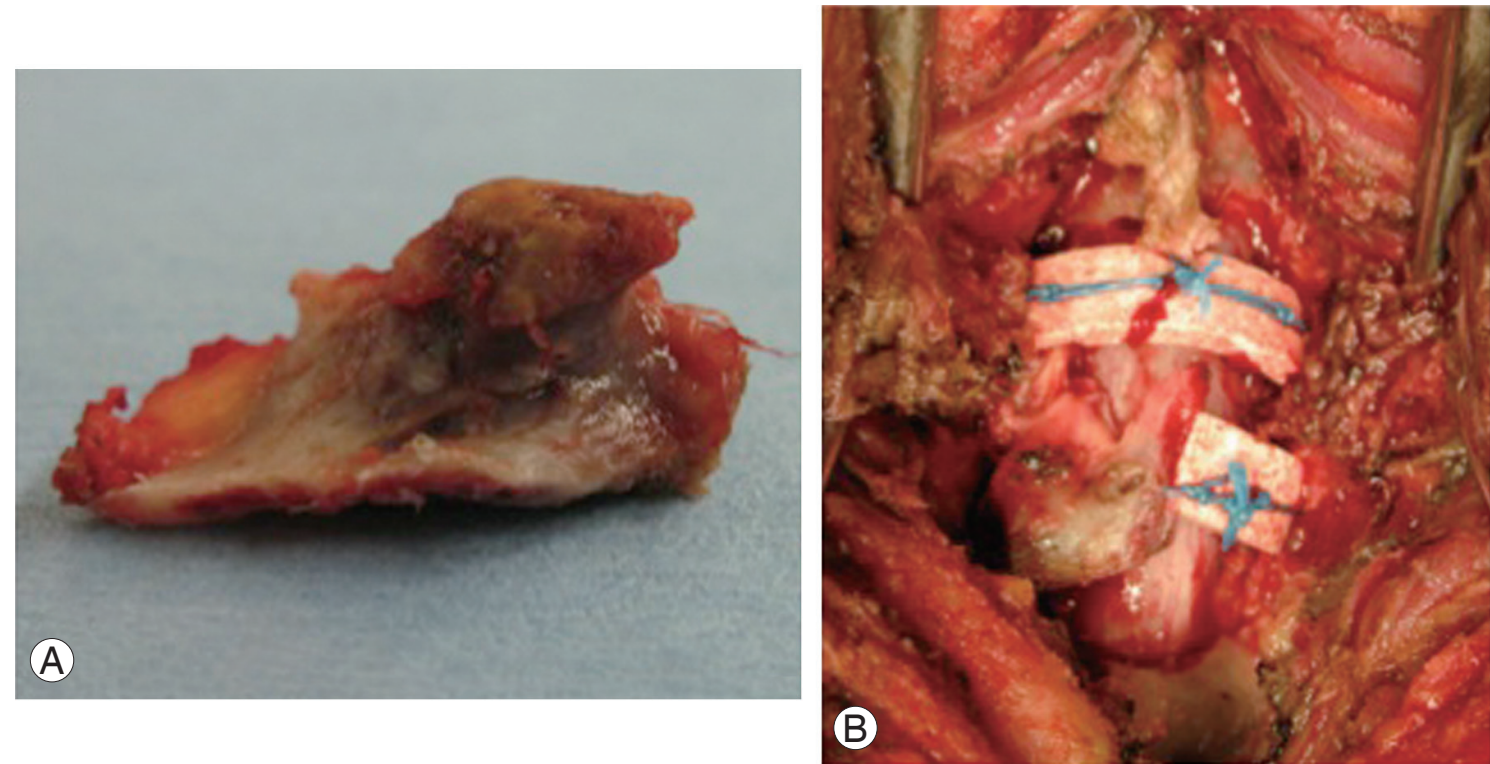

Fig. 3. (A) Macroscopic appearance of the tumor, with a cartilage cap and osseous stalk. (B) Intraoperative photograph is showing the reconstructed C5 and C6 laminas using hydroxyapatite spacers.
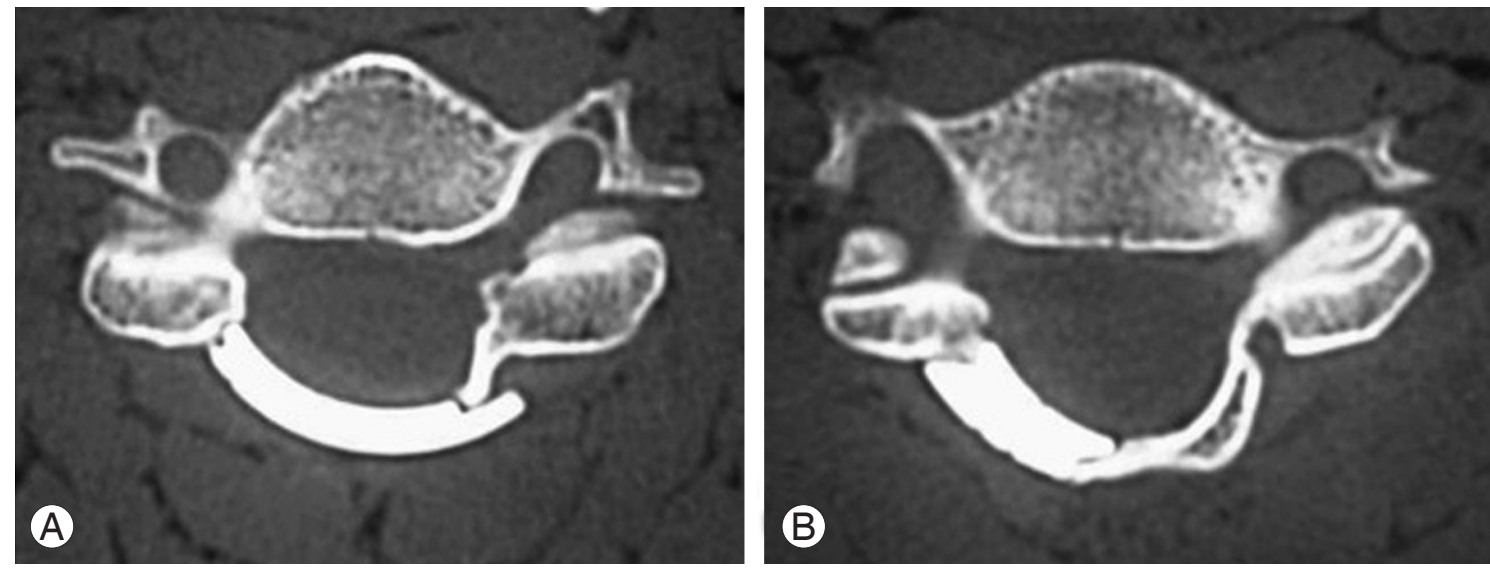

Fig. 4. Axial computed tomography scan at the C5 (A) and at the C6 (B) obtained at 1 year after surgery showing a sufficient enlargement of the spinal canal. 
quent displacement $[1,10]$. In 1907, Ochsner and Rothstein [11] reported the first case of cervical myelopathy in a patient with HME. To date, there have been more than fifty reported cases of cervical involvement with HME; Lotfinia et al. [1] presented a literature review with 48 summarized cases in 2010 [2-9]. Almost all of the reported cases underwent decompression surgery with tumor resection due to cervical myelopathy, or radiculopathy caused by spinal cord or nerve root compression of the intraspinal osteochondroma. On the other hand, Sakai et al. [12] reported middle-aged and elderly patients with

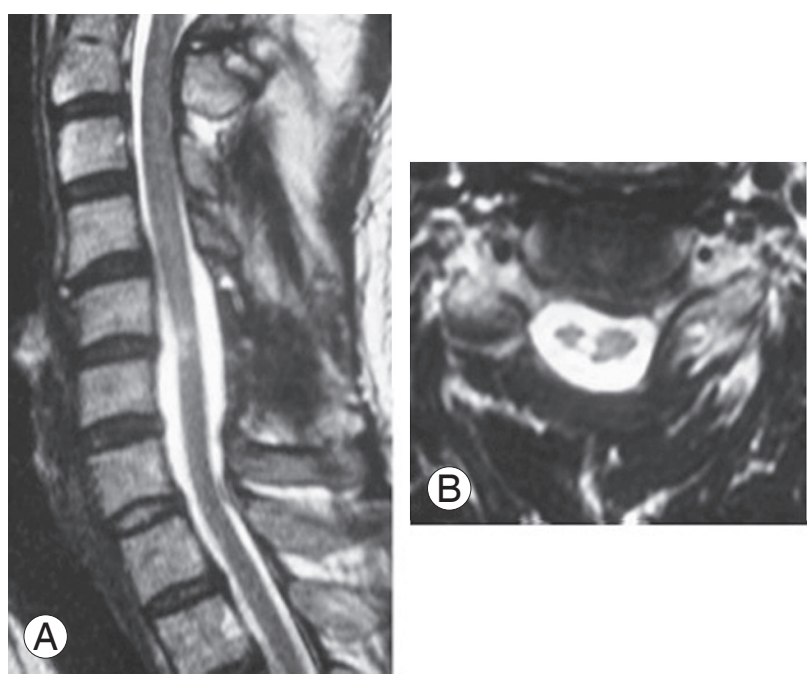

Fig. 5. Sagittal (A) and axial (B) T2-weighted magnetic resonance images obtained at 1 year after surgery showing a sufficient decompression of the spinal cord with a spontaneous regression of the herniated disc. However, the intramedullary lesion that identified as high intensity region remained. solitary spinal osteochondroma whose symptoms might be produced by degenerative changes of the cervical vertebrae at the segment of intraspinal osteochondroma. To the best of our knowledge, there have been no previous reports of cervical myelopathy caused by any degenerative changes of the vertebrae at the segment of existing intraspinal osteochondroma in patients with HME. The present case is the first reported case of HME with cervical compressive myelopathy caused by herniated disc at the segment of existing intraspinal osteochondroma. The patient's symptoms were clearly produced by appearance of herniated disc at the segment of existing intraspinal osteochondroma.

Surgical excision is the treatment of choice in all patients of symptomatic intraspinal osteochondroma and should be based on the extent and situation of the lesion. Complete removal of the lesion is essential to prevent recurrence $[1,10]$. Recurrence rates after resection are approximately $2 \%$, with a highly variable disease-free interval [10]. The most common surgical treatment for intraspinal osteochondroma is excision of the lesion by a decompressive laminectomy or hemilaminectomy because the intraspinal osteochondromas usually arise dorsally and dorsolaterally in the spinal column. However, a progressive postoperative kyphosis might occur with laminectomy for intraspinal osteochondroma because the lesion commonly appears in the second and third decade of life. On the other hand, laminoplasty had been developed to preserve the posterior structures of the spine and avoid the complications that would be accompanied
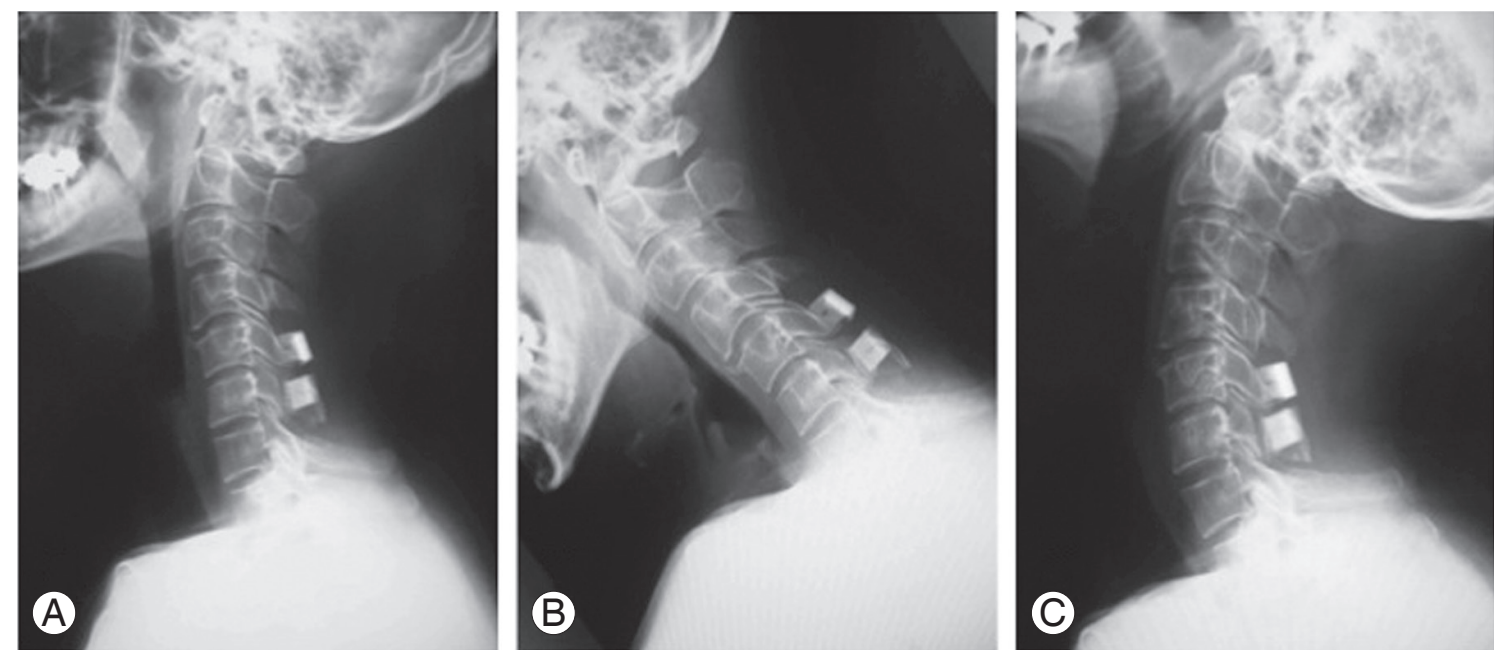

Fig. 6. Plain lateral radiographs showed no development of the cervical kyphosis (A) and flexion-extension radiographs revealed no segmental instability of the affected levels $(\mathbf{B}, \mathbf{C})$ at the three years after surgery. 
with laminectomy [13]. In 2000, Oga et al. [14] described a 13-year-old boy of intraspinal osteochondroma with HME, who could be successfully treated without a postoperative instability by a spinous process-splitting laminoplasty.

Although an anterior discectomy and spinal fusion (ASF), which was relatively simple and had acceptable surgical results, had been the most common surgical procedure for cervical disc herniation, some complications of ASF had been reported, especially on long-term follow-up. On the other hand, several reports had demonstrated spontaneous regressions of the herniated disc in the cervical spine, as well as in the lumbar spine [15-17]. Yoshida et al. [18] reported good clinical results of laminoplasty for cervical myelopathy caused by disc herniation with developmental canal stenosis. In the literature, a regression of the size of the herniated disc was noted in 15 of the 20 patients (75\%) who underwent laminoplasty. At the present, laminoplasty without removal of herniated discs has been a useful procedure for cervical compressive myelopathy caused by disc herniation.

The present case demonstrated cervical compressive myelopathy caused by intraspinal osteochondroma and disc herniation at the same segment. The osteochondroma might be able to be excised by hemilaminectomy of the $\mathrm{C} 5$, taking the size and location of the lesion into consideration. However, a bilateral posterior decompression or an additional anterior discectomy and fusion were necessary to complete decompression of the spinal cord because the herniated disc was on the opposite side. Thus, an open-door laminoplasty with excision of the tumor, which could be reconstructed the posterior structures of the spine, was performed in the present case. The herniated disc regressed spontaneously after the surgery, as well as in the previous reported cases with spontaneous regressions of cervical herniated disc. Consequently, a good surgical result was obtained for the present case without recurrence of the tumor and developing postoperative segmental instability in the cervical spine.

In conclusion, laminoplasty with excision of tumor, which could reconstruct the posterior structures of the spine, and without removal of herniated disc is a useful surgical procedure for compressive myelopathy caused by intraspinal osteochondroma arising from the posterior structures of the spine and disc herniation at the same segment.

\section{Conflict of Interest}

No potential conflict of interest relevant to this article was reported.

\section{References}

1. Lotfinia I, Vahedi P, Tubbs RS, Ghavame M, Meshkini A. Neurological manifestations, imaging characteristics, and surgical outcome of intraspinal osteochondroma. J Neurosurg Spine 2010;12:474-89.

2. Aldea S, Bonneville F, Poirier J, Chiras J, George B, Carpentier A. Acute spinal cord compression in hereditary multiple exostoses. Acta Neurochir (Wien) 2006;148:195-8.

3. Aniba K, Aldea S, Gaillard S. Cervical cord compression by hereditary multiple exostosis: case report and review of literature. Neurochirurgie 2011;57:85-7.

4. Burki V, So A, Aubry-Rozier B. Cervical myelopathy in hereditary multiple exostoses. Joint Bone Spine 2011;78:412-4.

5. Ezra N, Tetteh B, Diament M, Jonas AJ, Dickson P. Hereditary multiple exostoses with spine involvement in a 4-year-old boy. Am J Med Genet A 2010;152:1264-7.

6. Patel A, Thacker MM. Cervical spinal canal compromise in a 14-year-old girl with hereditary multiple exostoses. Pediatr Radiol 2010;40 Suppl 1:S158.

7. Roach JW, Klatt JW, Faulkner ND. Involvement of the spine in patients with multiple hereditary exostoses. J Bone Joint Surg Am 2009;91:1942-8.

8. Tahasildar N, Sudesh P, Goni V, Tripathy SK. Giant osteochondroma of axis in a child with multiple hereditary exostoses: case report and review of literature. J Pediatr Orthop B 2012;21:280-5.

9. Zaijun L, Xinhai Y, Zhipeng W, et al. Outcome and prognosis of myelopathy and radiculopathy from osteochondroma in the mobile spine: a report on 14 patients. J Spinal Disord Tech 2013;26:194-9.

10. Bess RS, Robbin MR, Bohlman HH, Thompson GH. Spinal exostoses: analysis of twelve cases and review of the literature. Spine (Phila Pa 1976) 2005;30:77480.

11. Ochsner EH, Rothstein T. XII. Multiple exostoses, including an exostosis within the spinal canal with surgical and neurological observations. Ann Surg 1907;46:608-16. 
12. Sakai D, Mochida J, Toh E, Nomura T. Spinal osteochondromas in middle-aged to elderly patients. Spine (Phila Pa 1976) 2002;27:E503-6.

13. Rhee JM, Basra S. Posterior surgery for cervical myelopathy: laminectomy, laminectomy with fusion, and laminoplasty. Asian Spine J 2008;2:114-26.

14. Oga M, Nakatani F, Ikuta K, Tamaru T, Arima J, Tomishige M. Treatment of cervical cord compression, caused by hereditary multiple exostosis, with laminoplasty: a case report. Spine (Phila Pa 1976) 2000;25:1290-2.

15. Kobayashi N, Asamoto S, Doi H, Ikeda Y, Matusmoto $\mathrm{K}$. Spontaneous regression of herniated cervical disc. Spine J 2003;3:171-3.

16. Matsumoto $\mathrm{M}$, Chiba $\mathrm{K}$, Ishikawa $\mathrm{M}$, Maruiwa $\mathrm{H}$,
Fujimura Y, Toyama Y. Relationships between outcomes of conservative treatment and magnetic resonance imaging findings in patients with mild cervical myelopathy caused by soft disc herniations. Spine (Phila Pa 1976) 2001;26:1592-8.

17. Mochida K, Komori H, Okawa A, Muneta T, Haro H, Shinomiya K. Regression of cervical disc herniation observed on magnetic resonance images. Spine (Phila Pa 1976) 1998;23:990-5.

18. Yoshida M, Tamaki T, Kawakami M, Hayashi N, Ando M. Indication and clinical results of laminoplasty for cervical myelopathy caused by disc herniation with developmental canal stenosis. Spine (Phila Pa 1976) 1998;23:2391-7. 